\title{
Clinical Impact of Histological Heterogeneity in the Metastatic Lymph Nodes of Patients with Colorectal Cancer
}

\author{
KAZUYOSHI HIRAYAMA, MAKOTO SUDOH, HIROKI SHIMIZU, SHINJI FURUYA, \\ YOSHIHIRO AKAZAWA, HIROSHI IINO, KENSUKE SHIRAISHI, HIDENORI AKAIKE, \\ NAOHIRO HOSOMURA, YOSHIHIKO KAWAGUCHI, HIDETAKE AMEMIYA, \\ HIROMITSU KAWAIDA, SHINGO INOUE, HIROSHI KONO, HIDEKI FUJII and DAISUKE ICHIKAWA
}

First Department of Surgery, Faculty of Medicine, University of Yamanashi, Yamanashi, Japan

\begin{abstract}
Aim: The purpose of this study was to evaluate the clinical impact of histological heterogeneity in patients with node-positive colorectal cancer (CRC). Patients and Methods: One hundred and twenty-nine patients who underwent curative surgical resection for histological node-positive CRC were enrolled. Patients were divided according to the histological heterogeneity in the primary lesion into p-hetero and p-homo groups. The p-hetero group was further divided according to histological heterogeneity in the metastatic lymph nodes into n-hetero and n-homo groups. Results: There were no significant differences between p-homo and p-hetero groups and between n-homo and n-hetero groups in prognosis. However, the recurrence-free survival rate of the n-homo group was significantly lower than that of the n-hetero group in the N2 category. Conclusion: Histological heterogeneity in metastatic lymph nodes may be useful for predicting prognosis, and prognosis in those with histological heterogeneity in a metastatic lymph node is not necessarily poor, even in those of the N2 category.
\end{abstract}

Colorectal cancer (CRC) is a common malignancy worldwide. As in Western countries, CRC has recently increased in many Asian countries in association with the westernization of lifestyle and diet. Recent advances in chemotherapeutics and molecular-targeted treatments have significantly improved the prognoses of patients with CRC. However, patients with advanced CRC frequently have tumor regrowth during intensive chemotherapy or experience relapse even after curative surgery with adjuvant

Correspondence to: Daisuke Ichikawa, MD, First Department of Surgery, Faculty of Medicine, University of Yamanashi, 1110 Shimokato, Chuo 4093898, Yamanashi, Japan. Tel: +81552737390 , Fax: +81 552737390, e-mail: dichikawa@yamanashi.ac.jp

Key Words: Histological heterogeneity, colorectal cancer, metastasis. chemotherapies. Therefore, the prognosis of patients with advanced CRC is by no means satisfactory.

Although the mechanism underlying the development of chemoresistance remains unclear, cancer stem cells have an integral role in treatment failure during chemotherapy. In addition, intra-tumor diversity has an important role in chemotherapeutic resistance, and intra-tumor heterogeneity causes resistance to treatment $(1,2)$. Recent comprehensive genetic analyses revealed that tumors constantly evolve in primary and metastatic lesions, which correlates with malignant potential such as invasion, metastasis, and treatment resistance. Metastatic lesions frequently develop from a sub-clone of the primary lesions but some metastatic lesions consist of multiple sub-clones with different mutational profiles $(3,4)$. In clinical practice, histological heterogeneity is recognized in various types of cancers (58 ), which correlates with specific clinicopathological features such as lymph node metastasis and poor prognosis (5-10).

In this study, we investigated the correlation between histological heterogeneity and other clinicopathological findings and evaluated the clinical impact of histological heterogeneity in patients with node-positive CRC.

\section{Materials and Methods}

Patients and clinicopathological features. A total of 388 patients with CRC underwent curative surgical resection at the University of Yamanashi Hospital between January 2008 and December 2014. All surgical procedures were in accordance with the Japanese Society for Cancer of the Colon and Rectum Guidelines (11). One hundred and twenty-nine patients with histological node-positive CRC who underwent curative resection with lymph node dissection were enrolled. Patients with distant metastases and multiple primary cancers were excluded. Patients treated with preoperative chemotherapy were also excluded. Demographic, clinical, and pathological features were obtained by reviewing medical records. The macroscopic and microscopic classifications of CRC were based on the 8th edition of the Union for International Cancer Control classification (12).

Postoperative adjuvant chemotherapy was performed in all patients except those refusing consent. Chemotherapy regimens 
Table I. Clinicopathological characteristics of 129 patients with nodepositive colorectal cancer.

\begin{tabular}{lc}
\hline Variable & Value \\
\hline Age, years & \\
$\quad$ Mean (range) & $69(24-85)$ \\
Gender, $\mathrm{n}(\%)$ & $80(62.0)$ \\
$\quad$ Male & $49(38.0)$ \\
Female & \\
ASA-PS, n (\%) & $25(19.4)$ \\
1 & $91(70.5)$ \\
2 & $13(10.1)$ \\
3 & $110(85.3)$ \\
Major histological type, n (\%) & $19(14.7)$ \\
Differentiated & \\
Undifferentiated & $39(30.2)$ \\
Location, n (\%) & $90(69.8)$ \\
Right-sided & \\
Left-sided & $50(10-120)$ \\
Tumor size, mm & \\
Mean (range) & $97(75.2)$ \\
UICC T category, n (\%) & $32(24.8)$ \\
$1-3$ & $94(72.9)$ \\
4 & $35(27.1)$ \\
UICC N category, n (\%) & \\
1 &
\end{tabular}

UICC: Union for International Cancer Control, ASA-PS: American Society of Anesthesiologists Physical Status.

were selected according to Japanese guidelines. The follow-up program consisted of a physical examination, hematological and blood chemistry panels, and serum tumor markers, such as carcinoembryonic antigen (CEA) and carbohydrate antigen 19-9 (CA19-9), every 3 months. Computed tomography (CT) from chest to pelvis was performed at least every 6 months. The study was approved by the Ethical Committee of Yamanashi University (approval no. 1899). Because recurrences were treated by various therapeutic options, such as surgical resection, radiation, or chemotherapy, recurrence-free survival (RFS) rate was investigated.

Histological heterogeneity of primary lesions and metastatic lymph nodes. Histological type was categorized into two groups: differentiated (well or moderately differentiated tubular adenocarcinoma) and undifferentiated (mucinous or poorly differentiated adenocarcinoma). Patients were classified into two groups depending on the tissue composition of the primary lesion: Homogenous histological type (p-homo) and heterogeneous histological type (p-hetero). The patients with heterogeneous histological primary tumors (p-hetero) were further classified into two sub-groups depending on the histological composition of the metastatic lymph nodes (n-homo and n-hetero).

Statistical analysis. Chi-squared and Fisher's exact probability tests for categorical variables and the Mann-Whitney $U$-test for unpaired data for continuous variables were performed to compare the clinicopathological characteristics between two groups. The
Table II. Clinicopathological characteristics of the group with homogeneous histological type in the primary lesion (p-homo) and that with heterogeneous histological type in the primary lesion ( $p$-hetero).

\begin{tabular}{lcc}
\hline Variable & $\begin{array}{c}\mathrm{p}-\text { Homo } \\
(\mathrm{n}=51,39.5 \%)\end{array}$ & $\begin{array}{c}\mathrm{p}-\text { Hetero } \\
(\mathrm{n}=78,60.5 \%)\end{array}$ \\
\hline Age, years & & \\
$\quad$ Mean (range) & $70(39-85)$ & $67(24-85)$ \\
Gender , n (\%) & & \\
$\quad$ Male & $32(62.7)$ & $48(61.5)$ \\
Female & $19(37.3)$ & $30(38.5)$ \\
ASA-PS, n (\%) & & \\
1 & $13(25.5)$ & $12(15.4)$ \\
2 & $33(64.7)$ & $58(74.4)$ \\
3 & $5(9.8)$ & $8(10.3)$ \\
Major histological type, $\mathrm{n}(\%)$ & & \\
$\quad$ Differentiated & $49(96.1)$ & $61(78.2)$ \\
$\quad$ Undifferentiated & $2(3.9)$ & $17(21.8)$ \\
Location, $\mathrm{n}(\%)$ & & \\
$\quad$ Right-sided & $11(21.6)$ & $28(35.9)$ \\
Left-sided & $40(78.4)$ & $50(64.1)$ \\
Tumor size, mm & & \\
$\quad$ Mean (range) & $50(10-110)$ & $49(15-120)$ \\
UICC T category, $\mathrm{n}(\%)$ & & \\
$1-3$ & $39(76.5)$ & $58(74.4)$ \\
4 & $12(23.5)$ & $20(25.6)$ \\
UICC N category, n (\%) & & \\
1 & $34(66.7)$ & $60(76.9)$ \\
2 & $17(33.3)$ & $18(23.1)$ \\
\hline
\end{tabular}

UICC: Union for International Cancer Control, ASA-PS: American Society of Anesthesiologists Physical Status.

cumulative survival rates were calculated using the Kaplan-Meier method, and the log-rank test was used for assessment of differences between prognostic factors. A $p$-value of less than 0.05 was considered significant.

\section{Results}

Patient characteristics and histological heterogeneity. The characteristics of 129 patients with node-positive CRC are summarized in Table I. The patients included 80 men and 49 women ranging in age from 24 to 85 years (median 69). Among the 129 patients, 51 patients (39.5\%) had primary lesions with a homogenous histological type (p-homo), and 78 patients $(60.5 \%)$ had lesions with various patterns of histological heterogeneity ( $p$-hetero), in which the major histological type was differentiated in 61 patients and undifferentiated in 17 patients. There were no significant differences between $\mathrm{p}$-homo and p-hetero groups in the various clinicopathological factors, except for the major histological type and tumor location. The frequency of differentiated major histological type was significantly higher in the p-homo group than in the p-hetero group. The 

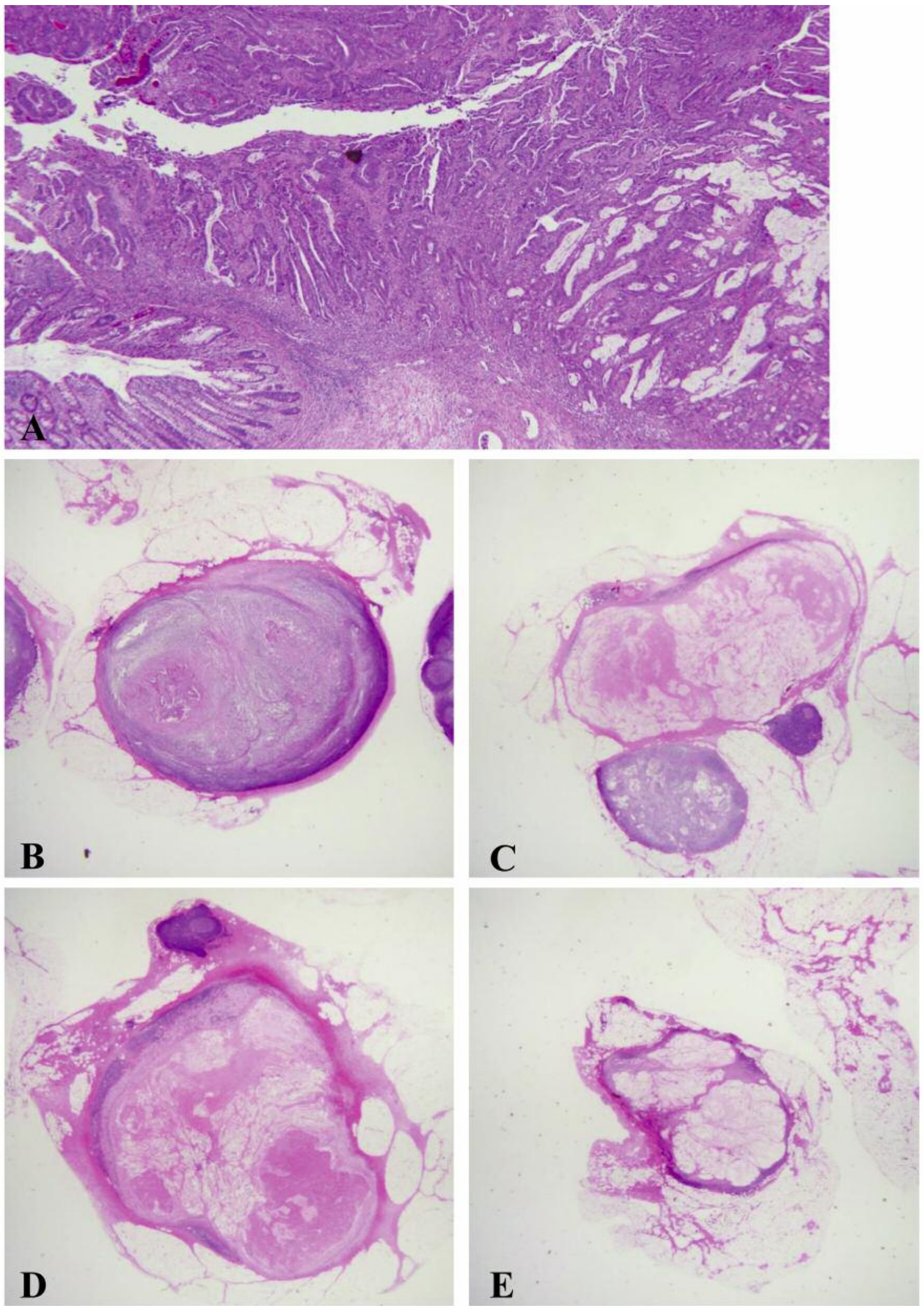

Figure 1. Histology of the primary lesion and metastatic lymph nodes. There were four metastatic lymph nodes in a patient with colorectal cancer with heterogeneous histological type in metastatic lymph nodes. A: Primary lesion, B: metastatic lymph node of differentiated type, $C$-E: metastatic lymph node of undifferentiated type. Original magnification, A: $\times 40, B-E: \times 12.5$. 


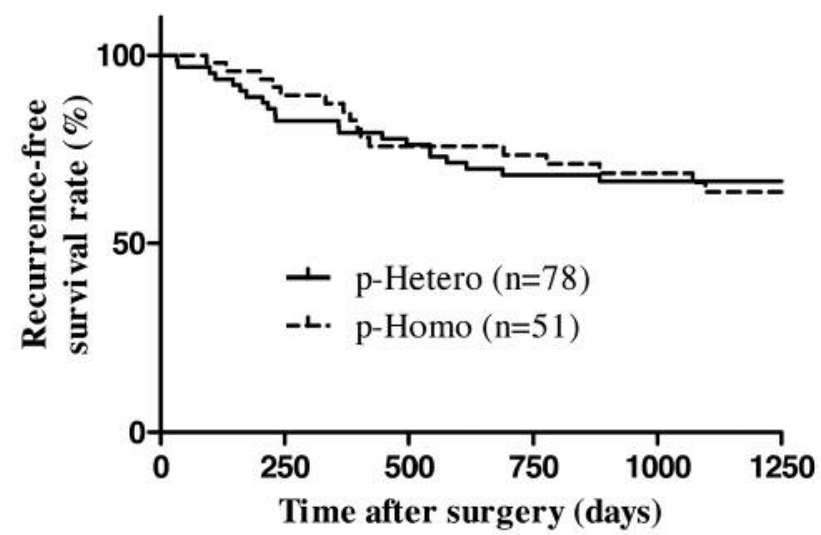

Figure 2. Comparison of recurrence-free survival by Kaplan-Meier survival method between the group with homogeneous histological type in the primary lesion (p-homo) and that with heterogeneous histological type in the primary lesion (p-hetero). There was no significant difference in the recurrence-free survival rate of the two groups.

frequency of right-sided tumors with histological heterogeneity was higher for primary lesions, but the difference was not significant (Table II).

Of the 78 patients in the p-hetero group, 27 had histological heterogeneity in their positive nodes, and histology was homogenous in the lymph nodes of the remaining 51 patients even though multiple nodes were involved. Histology was mostly homogenous within each positive node (Figure 1). There were no significant differences between the $\mathrm{n}$-homo and $\mathrm{n}$-hetero groups in terms of main histological type and other clinicopathological factors, except for the Union for International Cancer Control $\mathrm{N}$ category: The frequency of N2 tumors was significantly higher in the n-hetero group than in the n-homo group (Table III).

Prognostic impact of histological heterogeneity. The mean and median follow-up durations were 1,176 and 1,290 days, respectively. Recurrences occurred in 19 and 26 patients in the p-homo and p-hetero groups, respectively. The RFS rates were significantly lower for those with more advanced $\mathrm{T}$-and $\mathrm{N}$ factors than for lower T-and $\mathrm{N}$-factors. However, there were no significant differences in any other clinicopathological factor including heterogeneity of primary tumors (Figures 2 and 3).

Further survival analysis of the p-hetero group revealed that the RFS rate of the n-homo group was slightly better than that of the n-hetero group, but not significantly so (Figure 4). In subgroup analyses according to $\mathrm{N}$ category, the RFS rate of the $n$-homo group was significantly lower than that of the n-hetero group for the N2 category, but there was no significant difference in the RFS rate between the n-homo and n-hetero groups for the N1 category (Figure 5).
Table III. Clinicopathological characteristics of the n-homo and n-hetero groups.

\begin{tabular}{lcc}
\hline Variable & $\begin{array}{c}\mathrm{n} \text {-Homo } \\
\mathrm{n}=51(65.4 \%) \\
\text { Value }\end{array}$ & $\begin{array}{c}\mathrm{n} \text {-Hetero } \\
\mathrm{n}=27(34.6 \%) \\
\text { Value }\end{array}$ \\
\hline $\begin{array}{cc}\text { Age, years } \\
\text { Mean (range) }\end{array}$ & $69(24-85)$ & $63(26-85)$ \\
Gender, $\mathrm{n}(\%)$ & & \\
Male & $31(60.8)$ & $17(63.0)$ \\
Female & $20(39.2)$ & $10(37.0)$ \\
ASA-PS, $\mathrm{n}(\%)$ & & \\
1 & $8(15.7)$ & $4(14.8)$ \\
2 & $37(52.5)$ & $21(77.8)$ \\
3 & $6(11.8)$ & $2(7.4)$ \\
Major histological type, $\mathrm{n}(\%)$ & & \\
Differentiated & $39(76.5)$ & $22(81.5)$ \\
Undifferentiated & $12(23.5)$ & $5(18.59$ \\
Location, $\mathrm{n}(\%)$ & & \\
Right-sided & $22(43.1)$ & $6(22.2)$ \\
Left-sided & $29(56.9)$ & $21(77.8)$ \\
Tumor size, mm & & \\
Mean (range) & & \\
UICC T category, $\mathrm{n}(\%)$ & $45(15-120)$ & $38(20-70)$ \\
$1-3$ & $39(76.5)$ & $19(70.4)$ \\
4 & $12(23.5)$ & $8(29.6)$ \\
UICC N category, $\mathrm{n}(\%)$ & & \\
1 & $45(88.2)$ & $15(55.6)$ \\
2 & $6(11.8)$ & $12(44.4)$ \\
\hline
\end{tabular}

n-homo: Homogeneous histological type in metastatic lymph nodes, nhetero: heterogeneous histological type in metastatic lymph nodes, UICC: Union for International Cancer Control, ASA-PS: American Society of Anesthesiolorists Physical Status.

\section{Discussion}

Although CRC sometimes comprises several histological subtypes, the influence of histological heterogeneity on disease progression and treatment responses remains controversial. Kotanagi et al. showed that carcinomas with histological heterogeneity in the primary lesion had a higher incidence of lymph node metastasis and a worse prognosis than those without histological heterogeneity (10), and histological heterogeneity correlates with lymphatic progression in various types of cancer (5-8). These findings prompted us to investigate the clinical impact of histological heterogeneity in patients with node-positive CRC.

The undifferentiated major histological type and rightsided location were more frequently observed in the p-hetero group. In general, most CRCs are differentiated, and undifferentiated adenocarcinoma is an independent prognostic factor. However, we analyzed only patients with node-positive, and the RFS of the p-hetero group, including those with undifferentiated histological type, was equivalent to that of the p-homo group. Proximal colon cancer, which 

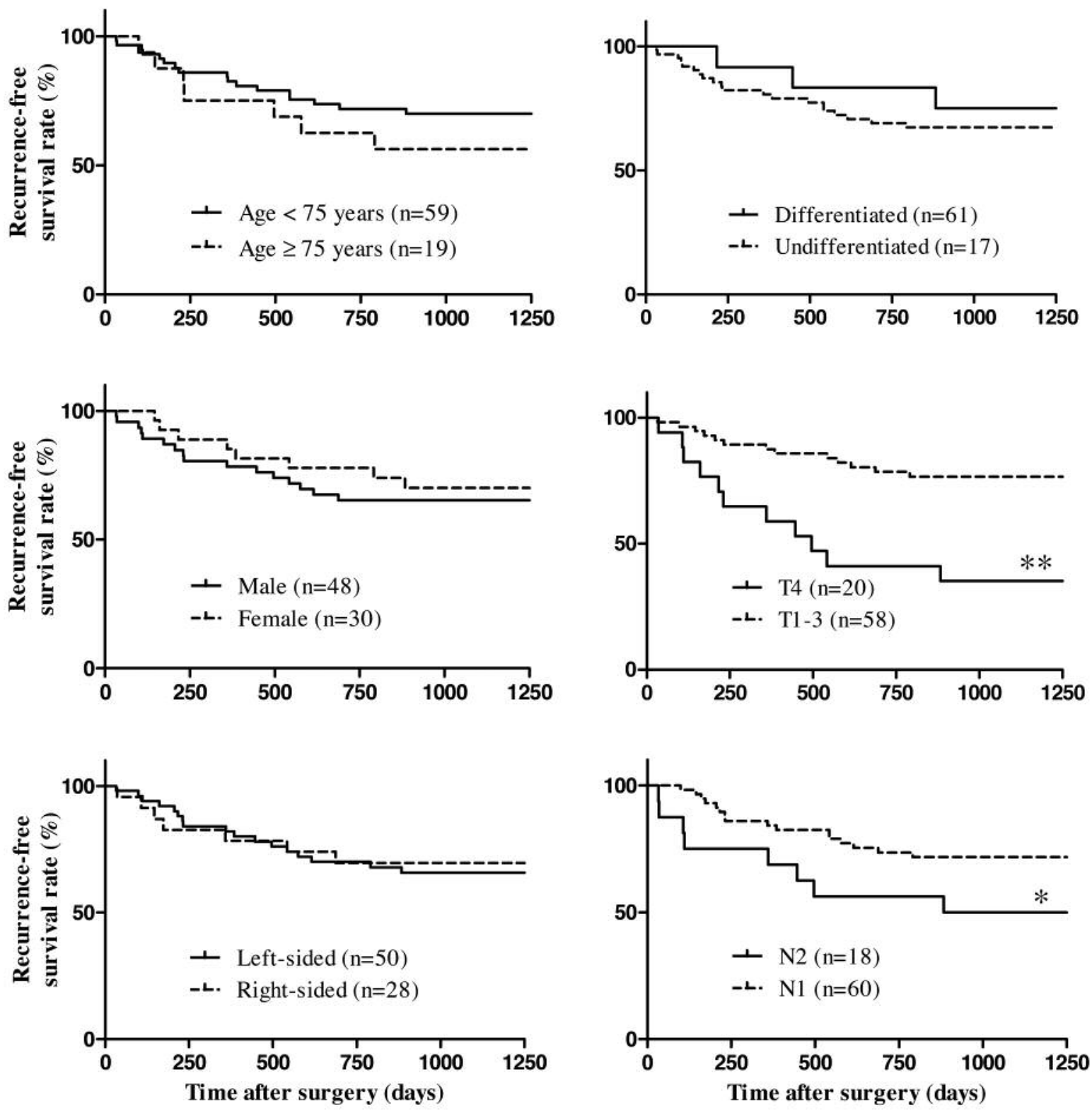

Figure 3. Comparison of recurrence-free survival by Kaplan-Meier survival method in the group with heterogeneous histological type in the primary lesion (p-hetero). The recurrence-free survival rates were significantly lower for patients with tumor of more advanced T-and $N$-factors than for those with lower $T$-and $N$-factors. There were no significant differences for any other clinicopathological factors. Significantly different at $* * p<0.01$ compared to T1-3 depth of tumor invasion and *p<0.05 compared to N1 by log-rank test.

comprises mainly poorly differentiated carcinoma, is associated with microsatellite instability and is associated with a good prognosis (13). Once lymph node metastasis occurs, the p-hetero group, which consists mainly of patients with tumor of undifferentiated histological type, may have similar prognosis to the p-homo group.

We analyzed the clinical impact of histological heterogeneity of the metastatic lymph node and its correlation to clinicopathological findings. In this study, 27 out of the 78 cases with a heterogeneous primary tumor (p-hetero) were formed from a single histological subtype in each positive node (Figure 1). Correlation analysis revealed that the more advanced $\mathrm{N} 2$ category was more frequent in the n-hetero group, which may affect prognosis. Therefore, we compared prognoses of both groups according to $\mathrm{N}$ category. Unexpectedly, for those in the $\mathrm{N} 2$ category, the $\mathrm{n}$-homo group 


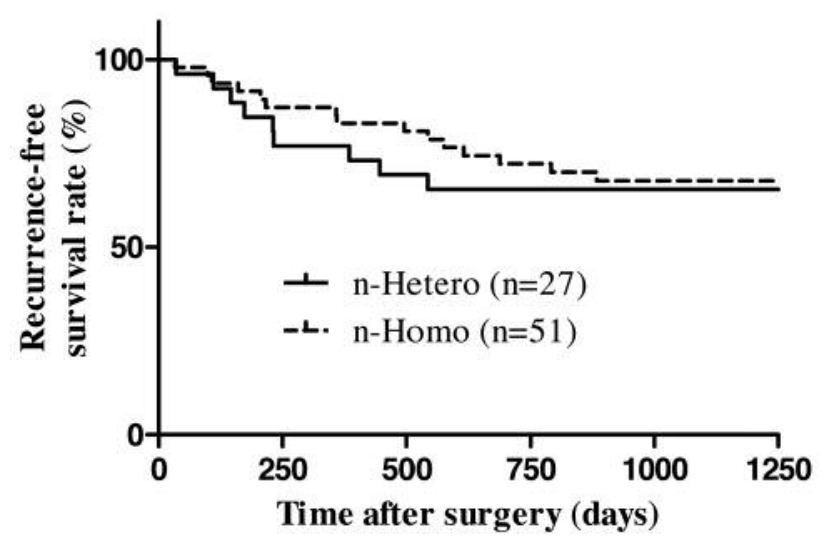

Figure 4. Comparison of recurrence-free survival by Kaplan-Meier survival method between the group with homogeneous histological type in metastatic lymph nodes ( $n$-homo) and that with heterogeneous histological type in metastatic lymph nodes (n-hetero). The recurrencefree survival rate of the n-homo group was slightly better than that of the n-hetero group, but there was no significant difference.

had a significantly lower RFS rate compared with the nhetero group, but there was no significant difference in rate between the groups for the N1 category. In the N2 category, the number of metastatic lymph nodes were defined as more than three. In this category, the metastatic nodes comprised histologically heterogenous metastases from different histological subtypes of primary lesion, and could therefore include e.g. one from differentiated and one from undifferentiated subtype, and two from undifferentiated subtype, making four metastases, defined as $\mathrm{N} 2$ in total. Therefore, the $\mathrm{N} 2 \mathrm{n}$-hetero group could be considered as simply arising from the sum of two different $\mathrm{N} 1$ subtypes.

Patients with advanced CRC, such as N2, have extremely poor prognosis. However, the development of intensive adjuvant multidrug chemotherapy may reduce the recurrence rate in this group. Our findings suggest that patients with $n$ hetero CRC may be candidates for intensive adjuvant multidrug chemotherapy, even those in the $\mathrm{N} 2$ category.

In conclusion, histological heterogeneity of metastatic lymph nodes may be useful for predicting prognosis, and the prognosis of patients with histological heterogeneity in a metastatic lymph node is not necessarily poor, even for those in the N2 category.

\section{References}

1 Burrell RA and Swanton C: Tumour heterogeneity and the evolution of polyclonal drug resistance. Mol Oncol 8: 10951111, 2014.

2 Bozic I and Nowak MA: Timing and heterogeneity of mutations associated with drug resistance in metastatic cancers. Proc Natl Acad Sci USA 111: 15964-15968, 2014.
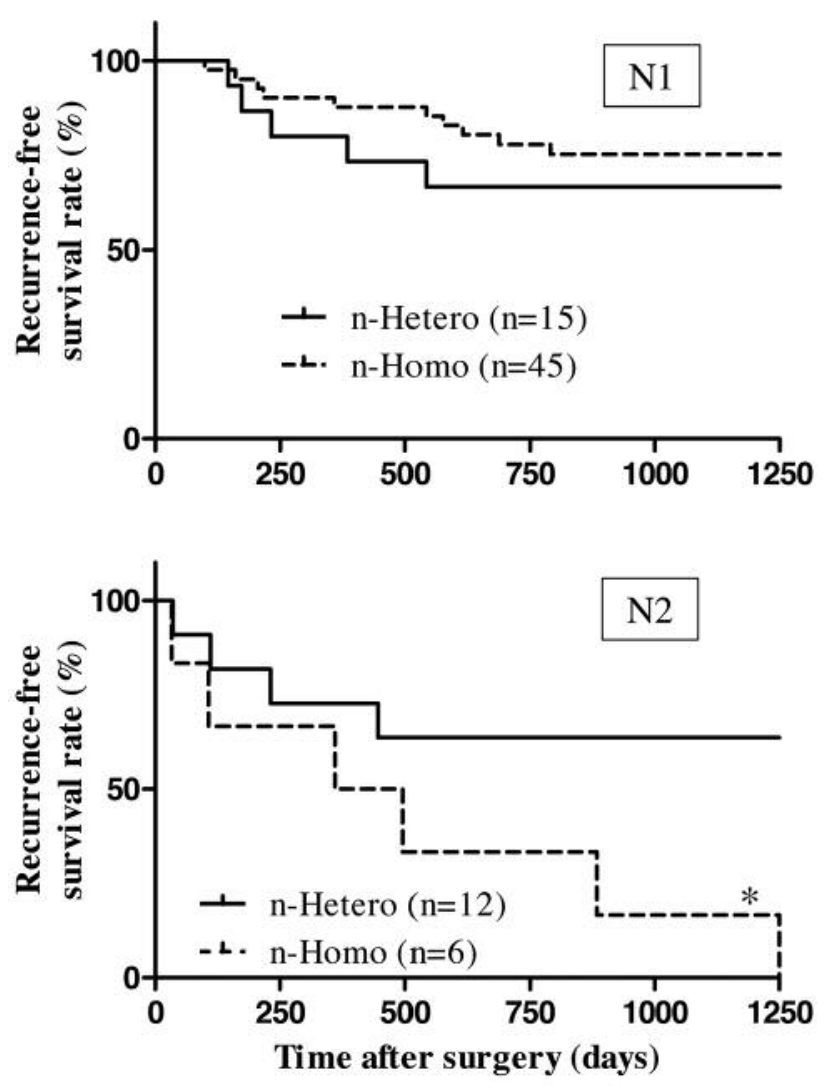

Figure 5. Comparison of survival curves by the Kaplan-Meier survival method between the group with homogeneous histological type in metastatic lymph nodes (n-homo) and that with heterogeneous histological type in metastatic lymph nodes (n-hetero). The recurrencefree survival rate of the $n$-homo group was significantly lower than that of the n-hetero group for those of the N2 category, but there was no significant difference in the rate between the n-homo and n-hetero groups in the N1 category. *Significantly different at $p<0.05$ compared to 2 of n-hetero group by log-rank test.

3 Jamal-Hanjani M, Wilson GA, McGranahan N, Birkbak NJ, Watkins TBK, Veeriah S, Shafi S, Johnson DH, Mitter R, Rosenthal R, Salm M, Horswell S, Escurero M, Matthews N, Rowan A, Chambers T, Moore DA, Turajlic S, Xu H, Lee SM, Forster MD, Ahmad T, Hiley CT, Abbosh C, Falzon M, Borg E, Marafioti BT, Lawrence D, Hayward M, Kolvekar S, Panagiotopoulos N, Janes SM, Thakrar R, Ahmed A, Blackhall F, Summers Y, Shah R, Joseph L, Quinn AM, Crosbie PA, Naidu B, Middleton G, Langman G, Trotter S, Nicolson M, Remmen H, Kerr K, Chetty M, Gomersall L, Fennell DA, Nakas A, Rathinam S, Anand G, Khan S, Russell P, Ezhil V, Ismail B, Irvin-Sellers M, Prakash V, Lester JF, Kornaszewska M, Attanoos R, Adams H, Davies H, Dentro S, Taniere P, O'Sullivan B, Lowe HL, Hartley JA, Iles N, Bell H, Ngai Y, Shaw JA, Herrero J, Szallasi Z, Schwarz RF, Stewart A, Quezada SA, Quesne L, Loo PV, Dive C, Hackshaw A and Swanton C: Tracking the evolution of non-small-cell lung cancer. N Engl J Med 376: 2109-2121, 2017. 
4 Abbosh C, Birkbak NJ, Wilson GA, Jamal-Hanjani M, Constantin T, Salari R, Le Quesne JL, Moore DA, Veeriah S, Rosenthal R, Marafioti T, Kirkizlar E, Watkins TBK, McGranahan N, Ward S, Martinson L, Riley J, Fraioli F, Bakir MA, Grönroos E, Zambrana F, Endozo R, Bi WL, Fennessy FM, Sponer N, Johnson D, Laycock J, Shafi S, Czyzewska-Khan J, Rowan A, Chambers T, Matthews N, Turajlic S, Hiley C, Lee SM, Forster MD, Ahmad T, Falzon M, Borg E, Lawrence D, Hayward M, Kolvekar S, Panagiotopoulos N, Janes SM, Thakrar R, Ahmed A, Blackhall F, Summers Y, Hafez D, Naik A, Ganguly A, Kareht S, Shah R, Joseph L, Quinn AM, Crosbie PA, Naidu B, Middleton G, Langman G, Trotter S, Nicolson M, Remmen H, Kerr K, Chetty M, Gomersall L, Fennell DA, Nakas A, Rathinam S, Anand G, Khan S, Russell P, Ezhil V, Ismail B, Irvin-Sellers M, Prakash V, Lester JF, Kornaszewska M, Attanoos R, Adams H, Davies H, Oukrif D, Akarca AU, Hartley JA, Lowe HL, Lock S, Iles N, Bell H, Ngai Y, Elgar G, Szallasi Z, Schwarz RF, Herrero J, Stewart A, Quezada SA, Peggs KS, Loo PV, Dive C, Lin CJ, Rabinowitz M, Aerts HJWL, Hackshaw A, Shaw JA, Zimmermann BG, TRACERx consortium, PEACE consortium and Swanton C: Phylogenetic ctDNA analysis depicts early-stage lung cancer evolution. Nature 545: 446-451, 2017.

5 Shimizu H, Ichikawa D, Komatsu S, Okamoto K, Shiozaki A, Fujiwara H, Murayama Y, Kuriu Y, Ikoma H, Nakanishi M, Ochiai T, Kokuba Y, Kishimoto M, Yanagisawa A and Otsuji E: The decision criterion of histological mixed type in "T1/T2" gastric carcinoma - comparison between TNM classification and Japanese Classification of Gastric Cancer. J Surg Oncol 105: 800-804, 2012.

6 Lee G, Choi ER, Lee HY, Jeong JY, Ahn JH, Kim S, Bae J, Kim HK, Choi YS, Kim J, Zo J, Lee KS and Shim YM: Pathologic heterogeneity of lung adenocarcinoma: A novel pathologic index predicts survival. Oncotarget 7: 70353-70363, 2016.

7 Reid MD, Balci S, Ohike N, Xue Y, Kim GE, Tajiri T, Memis B, Coban I, Dolgun A, Krasinskas AM, Basturk O, Kooby DA, Sarmiento JM, Maithel SK, El-Rayes BF and Adsay V: Ampullary carcinoma is often of mixed or hybrid histologic type: an analysis of reproducibility and clinical relevance of classification as pancreatobiliary versus intestinal in 232 cases. Mod Pathol 29: 1575-1585, 2016.
8 Tu SM, Bilen MA, Hess KR, Broaddus RR, Kopetz S, Wei C, Pagliaro LC, Karam JA, Ward JF, Wood CG, Rao P, Tu ZH, General R, Chen AH, Nieto YL, Yeung SC, Lin SH, Logothetis CJ and Pisters LL: Intratumoral heterogeneity: Role of differentiation in a potentially lethal phenotype of testicular cancer. Cancer 122: 1836-1843, 2016.

9 Büttner J, Jöhrens K, Klauschen F, Hummel M, Lenze D, Saeger $\mathrm{W}$ and Lehmann A: Intratumoral morphological heterogeneity can be an indicator of genetic heterogeneity in colorectal cancer. Exp Mol Pathol 104: 76-81, 2018.

10 Kotanagi H, Fukuoka T, Shibata Y, Yoshioka T, Aizawa O and Koyama K: The clinical significance of regional variations in histologic differentiation within carcinomas of the colorectum. Surg Today 23: 407-411, 1993.

11 Watanabe T, Itabashi M, Shimada Y, Tanaka S, Ito Y, Ajioka Y, Hamaguchi T, Hyodo I, Igarashi M, Ishida H, Ishihara S, Ishiguro M, Kanemitsu Y, Kokudo N, Muro K, Ochiai A, Oguchi M, Ohkura Y, Saito Y, Sakai Y, Ueno H, Yoshino T, Boku N, Fujimori T, Koinuma N, Morita T, Nishimura G, Sakata Y, Takahashi K, Tsuruta O, Yamaguchi T, Yoshida M, Yamaguchi N, Kotake K and Sugihara K: Japanese Society for Cancer of the Colon and Rectum (JSCCR) Guidelines 2014 for treatment of colorectal cancer. Int J Clin Oncol 20: 207-239, 2015.

12 Brierley JD, Gospodarowicz MK and Wittekind C (eds.): TNM Classification of Malignant Tumours, Eighth Edition. John Wiley \& Sons, Ltd. 2017.

13 Imai Y: Poorly differentiated adenocarcinoma of the colon: Subsite location and clinicopathologic features. Int J Colorectal Dis 30: 187-196, 2015.
Received June 16, 2018

Revised June 29, 2018

Accepted July 4, 2018 